livraisons

d'Histoire

de l'Architecture

\section{Livraisons de l'histoire de l'architecture}

14 | 2007

Piscines

\title{
La piscine du palais de cristal à Metz : une " apocalypse joyeuse »
}

The swimming pool of the "palais de cristal" in Metz: a merry apocalypse

Der frohe Untergang der Badeanstalt Kristallpalast in Metz

\section{Christiane Pignon-Feller}

\section{(Q) OpenEdition}

\section{Journals}

Édition électronique

URL : http://journals.openedition.org/lha/428

DOI : $10.4000 /$ lha. 428

ISSN : 1960-5994

Éditeur

Association Livraisons d'histoire de l'architecture - LHA

Édition imprimée

Date de publication : 10 décembre 2007

Pagination : 69-79

ISSN : 1627-4970

Référence électronique

Christiane Pignon-Feller, «La piscine du palais de cristal à Metz : une " apocalypse joyeuse » », Livraisons de l'histoire de l'architecture [En ligne], 14 | 2007, mis en ligne le 10 décembre 2009, consulté le 04 mai 2019. URL : http://journals.openedition.org//ha/428 ; DOI : 10.4000//ha.428

Ce document a été généré automatiquement le 4 mai 2019

Tous droits réservés à l'Association LHA 


\title{
La piscine du palais de cristal à Metz : une « apocalypse joyeuse »
}

\author{
The swimming pool of the "palais de cristal" in Metz: a merry apocalypse \\ Der frohe Untergang der Badeanstalt Kristallpalast in Metz
}

\section{Christiane Pignon-Feller}

1 Lorsque, par le traité de Francfort, en 1871, Metz fut annexée au deuxième Reich allemand et fit partie du Reichsland Elsass-Lothringen, la ville, parcourue par les cours et les bras morts de la Moselle et de la Seille, venait tout juste de se doter d'un système moderne de captation d'eaux de source, de s'enrichir de vingt-six nouvelles fontaines publiques et d'ouvrir deux bains et lavoirs municipaux qui firent l'admiration des nouveaux occupants ${ }^{1}$. Le projet d'un "établissement balnéaire complet» était, malheureusement, resté lettre morte ${ }^{2}$. Trente ans plus tard, l'état sanitaire déplorable de la vieille ville fut l'une des multiples causes qui accélérèrent la décision d'abattre les remparts obsolètes qui l'enserraient et de fonder ex novo, sur des terrains limités rachetés par la municipalité à l'autorité militaire, une nouvelle ville ${ }^{3}$.

2 Celle-ci fut planifiée selon les concepts les plus modernes de la science naissante de l'urbanisme et mêla harmonieusement les théories progressistes aux principes culturalistes. Si dans cette ville à croissance interne diversifiée, les questions de voirie et la situation, la fonction, la forme des équipements publics et semi publics étaient réglées de manière magistrale, si les initiatives privées apportaient une bonne part des équipements de culture et de loisirs, on pouvait cependant déplorer l'absence d'un établissement moderne capable de suppléer les établissements du XIX siècle, les écoles de natation, les Wannenbäder privés, le premier Brausebad ${ }^{4}$ public et les bains de rivière ${ }^{5}$.

3 Ce n'est pas que la municipalité se désintéressât de la question des établissements de bains puisque le bureau d'architecture de la ville avait acquis une série de recueils et d'ouvrages illustrés traitant des constructions balnéaires en Europe ${ }^{6}$. Mais, comme il était couramment admis que «la construction d'une piscine n'est jamais rentable financièrement et que son fonctionnement ne l'est que très rarement $»^{7}$, la municipalité avait évité d'accroître son endettement par la construction d'un établissement aussi peu 
lucratif déclaré cependant " œuvre de progrès nécessaire à la ville »8 et que les théories hygiénistes et les débuts du culte du corps mettaient à l'ordre du jour.

\section{Où la municipalité se décharge d'un devoir contraignant}

4 En 1906, un particulier se déclara acquéreur, dans la prestigieuse rue menant à la nouvelle gare en construction, d'une parcelle de 23 ares sur un îlot de la zone de constructions fermées ${ }^{9}$ afin d'y construire un établissement de bains. Au préalable il soumettait à la municipalité une esquisse de cahier des charges: l'établissement comporterait « deux bassins de natation ( $250 \mathrm{~m}^{2}$ pour hommes, $180 \mathrm{~m}^{2}$ pour dames), une soixantaine de baignoires (séparées en 3 classes), des bains électriques, des bains d'air chaud et de vapeur avec cabinet de massage et de repos, des bains médicinaux, des bains d'air et de soleil, un institut de gymnastique hygiénique, un inhalatoire $»^{10}$. De ces thermes antiques ressuscités il n'existait, aux dires du promoteur, que trente-sept exemples dans toute l'Allemagne. Sautant sur cette occasion inespérée qui la libérait d'une charge qu'elle ne pourrait remplir de sitôt, l'approvisionnement en eau étant jugé suffisant et la moralité de la population étant garantie par la séparation des sexes, la municipalité gela la parcelle (obligatoirement balnéaire) mais vota en compensation, à la demande de l'acquéreur, une clause de non concurrence qui engageait la ville à ne pas construire de piscine municipale pendant vingt-cinq ans ${ }^{11}$. Pressentant probablement les aléas de l'entreprise, ce premier promoteur se désista. Un an plus tard, la municipalité vendait à Johann Balassa, jeune architecte juif hongrois, né à Vienne en $1881^{12}$ et installé à Metz depuis 1903, la "parcelle balnéaire " rétrécie à 10 ares qui fut néanmoins augmentée de 4 ares supplémentaires ${ }^{13}$. C'est ainsi que, après avoir parsemé les communes avoisinantes de quelques immeubles inspirés des travaux viennois d'Otto Wagner et d'Adolf Loos, Balassa fut investi du devoir de construire un établissement sportif et hygiénique au cœur de la nouvelle ville, dans une zone d'habitat serré, sur un petit terrain meuble, non dégagé, comportant une partie approximativement carrée, de 30 mètres de façade. Ce carré se prolongeait par un appendice latéral en fond de parcelle, ce qui donnait à l'ensemble une forme particulièrement ingrate. À ces premières difficultés s'ajoutait l'obligation de se plier aux contraintes du nouveau règlement urbain très exigeant ${ }^{14}$. Celui-ci déterminait, dans la zone donnée, l'occupation de la parcelle ( $7 / 10^{e}$ du terrain, les espaces libres ne devant pas occuper moins de $\left.40 \mathrm{~m}^{2}\right)$, la longueur et la largeur des saillies autorisées, la hauteur du bâtiment (de 15 à 18 mètres), la hauteur du faîtage (pas plus de la moitié de la profondeur de la parcelle y compris la saillie de la corniche), l'inclinaison de la corniche (pas plus de $50^{\circ}$ ), le nombre d'étages « habitables » outre le rez-de-chaussée (quatre), le nombre d'escaliers, la hauteur des pièces $(2,80$ mètres $)^{15}$. En outre, dans cette nouvelle ville s'appliquait une politique esthétique volontariste qui n'admettait en principe que l'emploi de la pierre de taille.

Balassa releva tous ces défis avec brio par une approche inédite du cahier des charges dont il outrepassa même les termes dans un programme d'une étonnante liberté. Pour ce faire, il s'adjoignit la collaboration d'un entrepreneur de Sarreguemines Jean-Nicolas Dietsch (ill. 1), « spécialiste de béton armé, de constructions aériennes et souterraines de toutes sortes, de bâtiments industriels clés en main, de béton précontraint de tous les genres et de béton armé ${ }^{16}$ qui devint administrativement maitre d'ouvrage de la 
construction mais déclara laisser les pleins pouvoirs architecturaux, techniques et commerciaux à Balassa ${ }^{17}$.

III. 1 : Papier à en-tête de l'entreprise Dietsch montrant notamment la structure de la piscine, 1909

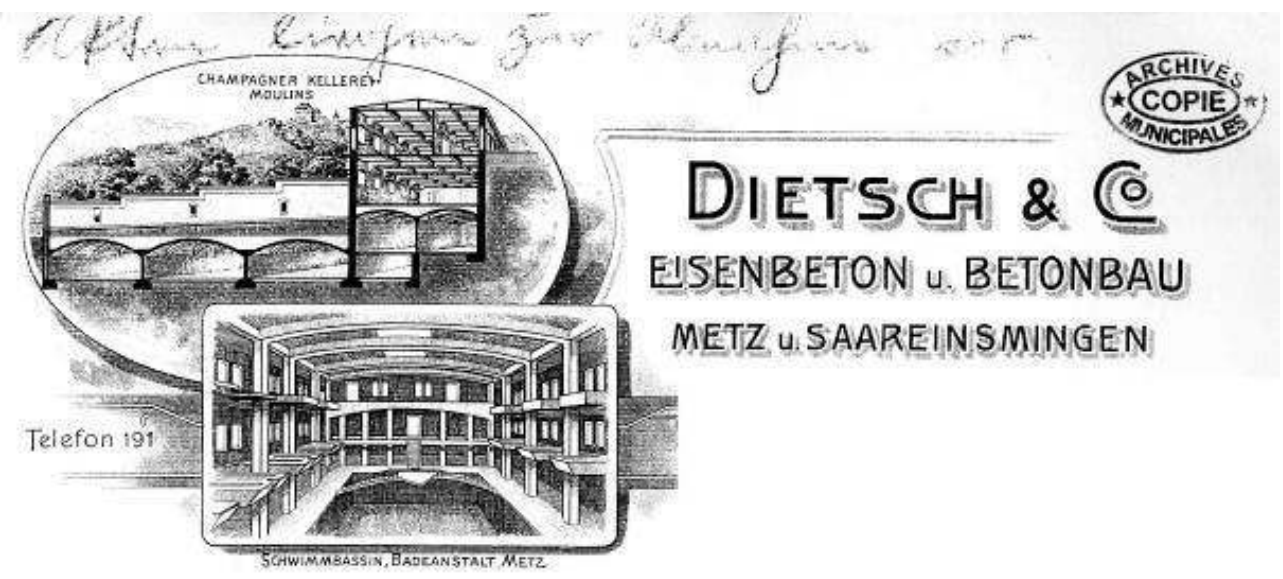

Arch. mun. Metz, PC 39 avenue Foch. Cl. de l'auteur

6 À chaque contrainte et à chaque difficulté, architecte et entrepreneur trouvèrent des solutions inusitées. En premier lieu, pour lever d'éventuelles réticences quant à l'utilité de l'établissement, ils firent donner une conférence vantant les bienfaits de la natation et en profitèrent pour expliquer les concepts techniques qui les guidaient ${ }^{18}$. La question de l'approvisionnement en eau fut réglée par le creusement de puits à fort débit qui rendait l'établissement indépendant du réseau municipal.

7 Le sous-sol étant meuble, ils creusèrent des fondations profondes de onze mètres et stabilisèrent le bâtiment par l'emploi de pilotis de béton armé. La parcelle étant relativement exiguë, ils développèrent leur établissement en hauteur ; mais la hauteur étant limitée ainsi que le nombre d'étages, ils multiplièrent les surfaces efficaces par un entresol et des mezzanines. Le terrain étant irrégulier, ils aménagèrent des cours intérieures, des escaliers et des dégagements par le fond de la parcelle. Enfin, un bassin de natation au rez-de-chaussée étant malaisé à éclairer, ils le hissèrent au deuxième étage afin de profiter d'un éclairage zénithal diurne. Les cours intérieures latérales leur permirent également de multiplier les baies du corps de bâtiment principal et de résoudre la question de l'ouverture des espaces intérieurs à l'air et à la lumière. Ils adhéraient ainsi au slogan «Licht und Luft », ligne de conduite de toutes les constructions de la nouvelle ville.

8 Les travaux de fondation commencèrent en novembre 1907. Malheureusement, Balassa avait négligé de suivre la procédure légale et ne déposa son dossier de demande d'autorisation de construire qu'en février 1908. Cette première entorse au règlement urbain fut le début d'une éprouvante lutte avec la municipalité qui, devant l'audace du projet, fit preuve d'une incroyable pusillanimité et ne cessa de retarder et de contrecarrer la construction du nouvel établissement. Ainsi, elle fit expertiser par un professeur suisse les calculs statiques de Dietsch, elle différa sine die l'agrément technique de la construction ou se déchargea sur des fonctionnaires intérimaires de la responsabilité de cet agrément, elle récusa l'esthétique du dessin d'élévation de l'architecte et lui demanda des dessins hors normes; le comble de la défiance fut atteint lorsqu'elle fit boucler l'ensemble du quartier par la police et les pompiers lors de la mise en eau du bassin (qui 
se fit sans dommages) ; enfin, lorsque les essais de chauffage provoquèrent des retombées de suies, une campagne de presse virulente s'éleva contre l'entreprise. Ces entraves municipales expliquent en partie le naufrage d'une des plus belles réalisations dont la ville s'était pourtant déchargée au profit du privé ${ }^{19}$.

\section{Le pari du béton armé et la prouesse de la piscine autoportante}

Si l'on avait déjà expérimenté à Metz les performances du béton armé, notamment dans son emploi en pilotis, en dalles et en porte-à-faux ${ }^{20}$, si les Moniergewölbe (voûtes Monier) étaient d'usage courant, si de nouveaux couvrements en dalles de verre plastique commençaient à se répandre, en revanche, la construction entièrement en béton armé était inédite. Non seulement l'architecte construisit en béton armé mais il se fit fort de n'employer aucune pierre de structure ou de parement, allant ainsi à l'encontre de la règle implicite d'esthétique urbaine ${ }^{21}$.

La révolution conceptuelle et technique de l'établissement résidait surtout dans l'idée incroyablement audacieuse de monter un bassin autoportant (selbstragend) de $250 \mathrm{~m}^{2}$ (d'une profondeur de $80 \mathrm{~cm}$ à 3 mètres) au deuxième étage (ill. 2) !

III. 2 : Coupe, dessin Hugues Duwig d'après planche 11/11 au 1/100e, février 1908

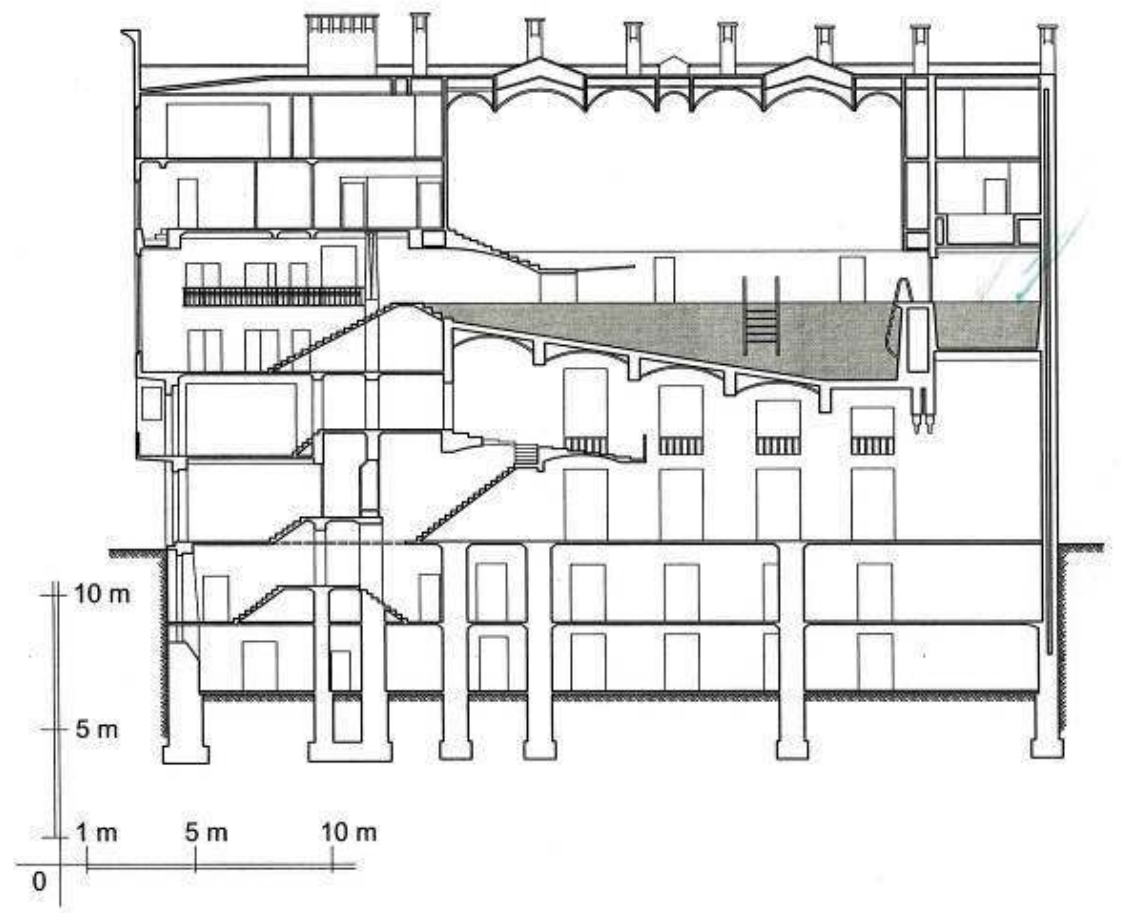

Arch. mun. Metz, 1 M 56

11 Cette idée montrait l'étonnant crédit que les concepteurs accordaient aux performances des matériaux nouveaux dont ils assumaient les risques en toute confiance. Tous les moyens des techniques modernes avaient d'ailleurs été mis en œuvre: bétonnière à tambour ininterrompu actionnée par un moteur électrique et servie par huit ouvriers qui 
déversait le béton dans des wagonnets, abattant ainsi le travail de vingt-cinq journaliers ${ }^{22}$ . Les impressionnantes fondations des deux niveaux de caves contenant la chaufferie à air pulsé et la salle des machines, l'épaisseur des éléments porteurs et des dalles, notamment la dalle supérieure supportant le réservoir d'eau montrent que l'on n'avait pas lésiné sur la qualité et la modernité des moyens. On ne fit pas davantage l'économie d'ascenseurs qui assuraient, avec des escaliers et des galeries, les nécessaires circulations ascendantes. La structure de l'établissement transparaissait nettement en façade avec ses pleins et ses vides et son réseau graphique, le ressaut central des bow-windows, ses balcons, son imposante corniche. La découpe des multiples baies rectangulaires, étroites et hautes attestait l'exigeante inspiration viennoise d'un Adolf Loos. Alors que les immeubles voisins déguisaient leur ossature sous des parements de pierres de taille et des décors historicistes ou pseudo régionalistes, Balassa osait affirmer la primauté de la structure sans la travestir, affirmant publiquement la collaboration de l'architecte et de l'ingénieur.

\section{Le scandale d'un programme et d'un plan au service de I'hédonisme}

Mêler les plaisirs des thermes antiques, les délices décadentes des villes d'eau hongroises, les folies viennoises de la Belle Époque et les jouissances des loisirs modernes, tel était le programme hédoniste de l'établissement qui détermina la complexité de son plan.

C'est dans les étages supérieurs que Balassa répondait aux données du programme sanitaire, sportif et hygiéniste préconisé. Le toit pour partie en terrasse était réservé à un solarium (bains d'air et de lumière) évidemment non mixte. Des bains sanitaires et médicaux [sic] et un atelier de photographe y trouvaient également place. Le bassin de natation du deuxième étage était de forme rectangulaire (ill. 3).

III. 3 : Plan du deuxième étage, dessin Hugues Duwig d'après planche 7/1 1

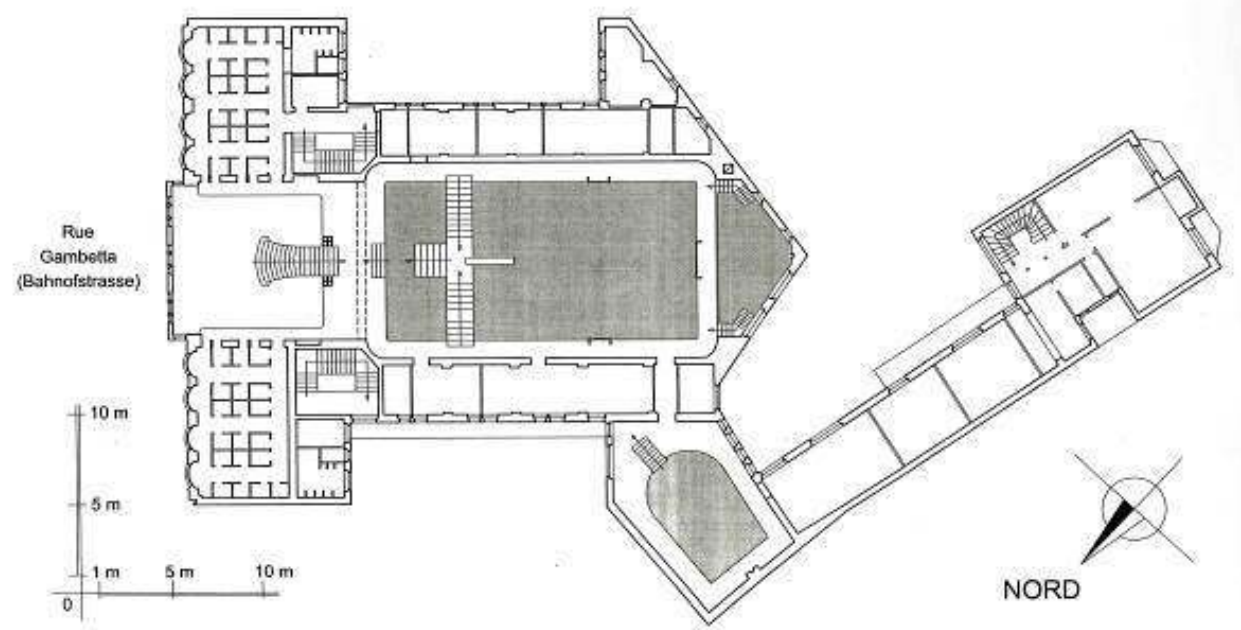

au $1 / 100^{e}$, février 1908

Arch. mun. Metz, $1 \mathrm{M} 56$

Un bassin d'eau chaude de forme triangulaire et moins profond prolongeait le bassin principal. Un petit bassin d'eau froide enfin rappelait le "parcours» des thermes 
antiques : tepidarium, caldarium et frigidarium. Un couvrement en verre coloré assurait l'éclairage diurne et des lustres électriques l'éclairage nocturne. Le grand bassin était entouré de cabines et de cellules qui prenaient jour sur les cours intérieures et où officiaient barbiers, coiffeurs, manucures, pédicures, dentistes ${ }^{23}$. Il était surmonté d'une mezzanine qui comportait des cabines de massages, des bains de vapeur, des bains électriques, des cabines à air chaud, des douches froides et des distributeurs automatiques. À l'étage supérieur se trouvaient des baignoires simples et doubles (de deuxième classe) et des bains familiaux. Toutes les cabines étaient pourvues de téléphones pour les communications locales ${ }^{24}$.

En bref, Balassa offrait aux Messins les inventions les plus modernes des soins du corps que les établissements des villes thermales avaient réinventées sur le modèle antique en les rebaptisant bains romains, bains irlandais, bains écossais ou bains allemands ${ }^{25}$.

Les autres étages de l'établissement, quant à eux, étaient réservés à des plaisirs moins hygiéniques ou sportifs. Champagne, vin, cuves de bière alimentant les «bars automatiques » remplissaient les caves qui leur étaient spécialement affectées. Au rez-dechaussée, un restaurant automatique (service debout ancêtre de nos fast-foods), un restaurant traditionnel, une salle de billard et une grande salle de cinéma avaient pris la place des boutiques, du glacier, du pressing initialement projetés. À l'entresol enfin régnait le Zillertal. Ce café-concert baptisé du nom d'une pittoresque vallée tyrolienne ${ }^{26}$ était une sorte de mystérieuse grotte labyrinthique parée de faux rochers et de stalagmites dans lesquels jouait un éclairage électrique coloré. Une estrade en bois en bouleau non écorcé était destinée à divers orchestres invités. Tout un cheminement secret menait à des cabinets particuliers sûrement moins bucoliques que le Zillertal !

Ce programme dionysiaque concrétisé par un plan minutieux ne pouvait évidemment que susciter des remous scandalisés dans une ville d'ordre militaire et dont les pratiques civiles étaient en général plus dévotes qu'hédonistes !

\section{Le défi du cristal et la provocation du décor palatial viennois}

Le Viennois ne pouvait achever son œuvre sans une dernière provocation : celle du décor. Ce palais d'Atlas et de Dionysos devint le champ expérimental de toutes les audaces décoratives. Plus fort que les concepteurs du Crystal Palace de Londres (1851), du Glaspalast de Munich (1853) ou du palais de cristal de Madrid (1887) qui n'étaient que de pâles métaphores, Balassa allait étonner le monde en proposant une métonymie de la transparence de l'eau et employer, pour décorer son chef-d'œuvre, du vrai cristal de Bohème (" echtem Krystall Böhmische Glaserei »). La tentation de l'imitation à bas prix étant rejetée avec hauteur ${ }^{27}$, les carreaux de cristal recouverts d'une couche de peinture irisée couvrirent non seulement les parois des bassins de natation mais le cristal de couleur en plaques et en cabochons irradia la totalité de la façade de ce palais qui ainsi mérita sa double appellation de Krystall Palast / Palais de cristal (ouvert aux autochtones francophones). La leçon de la noblesse des matériaux et leur emploi en plaques de surface lui venaient d'évidence de Vienne où elle avait été expérimentée par Otto Wagner ${ }^{28}$. La réalisation du travail fut, en revanche, confiée aux ateliers locaux Baudinet où Balassa convoqua en vain les responsables municipaux pour les persuader de la qualité de son 
travail. Il reste de cette façade un dessin aquarellé ponctué d'or (ill. 4) où, à la demande de la municipalité, l'architecte a tenté de reproduire sa coruscante invention.

\section{4 : Dessin aquarellé de la façade}

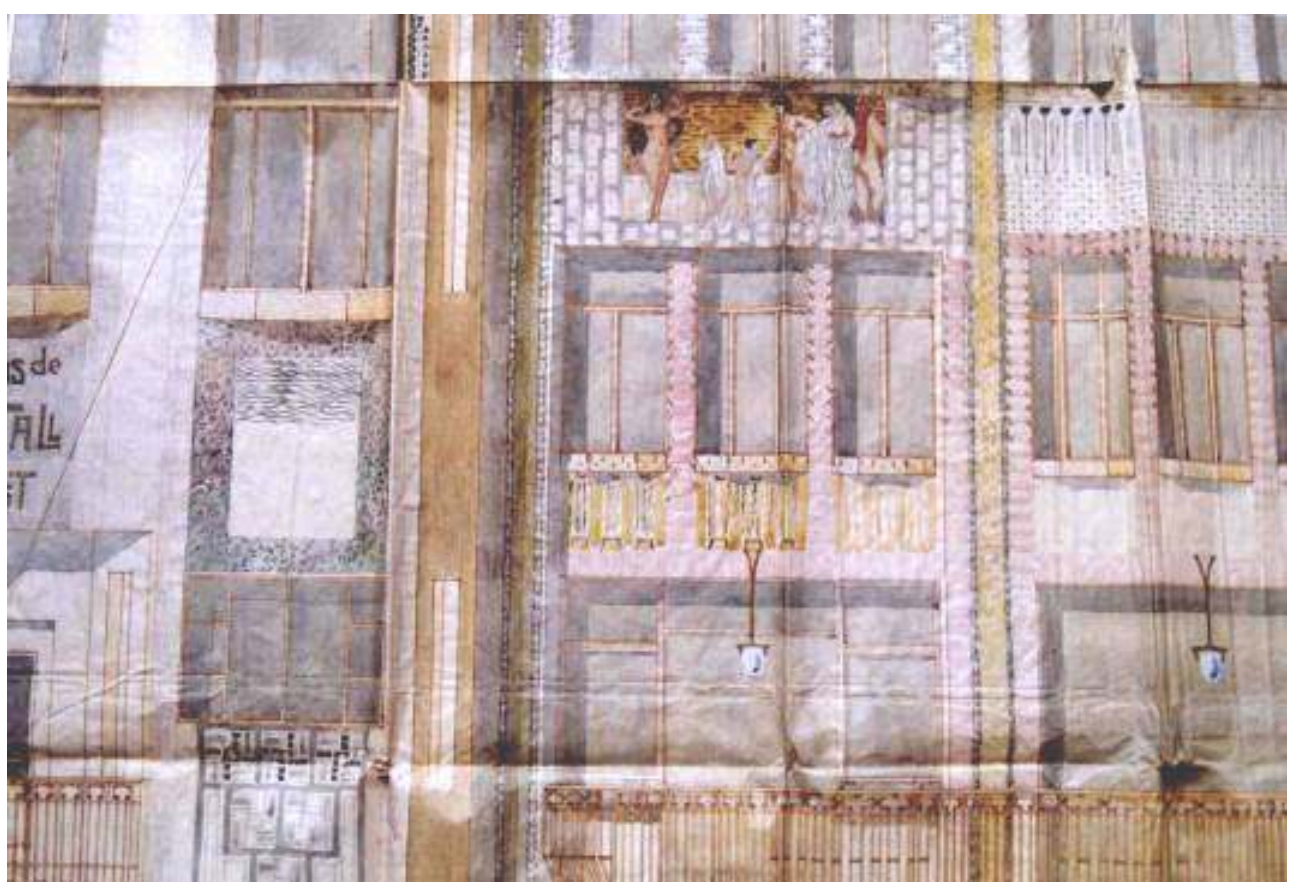

Arch. mun. Metz, 1M56. Cl. de l'auteur

19 Les motifs géométriques que l'on y voit rappellent autant les recherches graphiques de Josef-Maria Olbrich que celles de l'atelier viennois des arts appliqués appelés Wiener Werkstätte. Vienne et sa précieuse et trouble sensualité étaient encore sur la façade où deux panneaux en mosaïque donnaient le ton de l'établissement. Des baigneuses nues y étaient traitées dans une facture qui rappelait celle de Klimt et dans des attitudes inspirées par le sulfureux Egon Schiele ou Kolo Moser.

20 Vienne était enfin dans les détails; moins dans l'imitation assez populiste du Zillertal, où la bière coulait à flots et qui résonnait des flonflons d'orchestres bavarois, que dans le décor de ferronnerie, de stucs ${ }^{29}$ et de vitraux. On était loin des contorsions de l'Art nouveau! L'élégance austère et abstraite de la ligne s'inspirait encore de la Sécession viennoise. Et probablement aussi la couleur.

21 L'austérité du graphisme semblait, si l'on en croit les témoignages, compensée par la richesse des coloris. Certes, la carte postale colorisée qui nous reste de l'intérieur de la piscine semble un rien bariolée (ill. 5). 
III. 5 : Le bassin de natation du palais de cristal

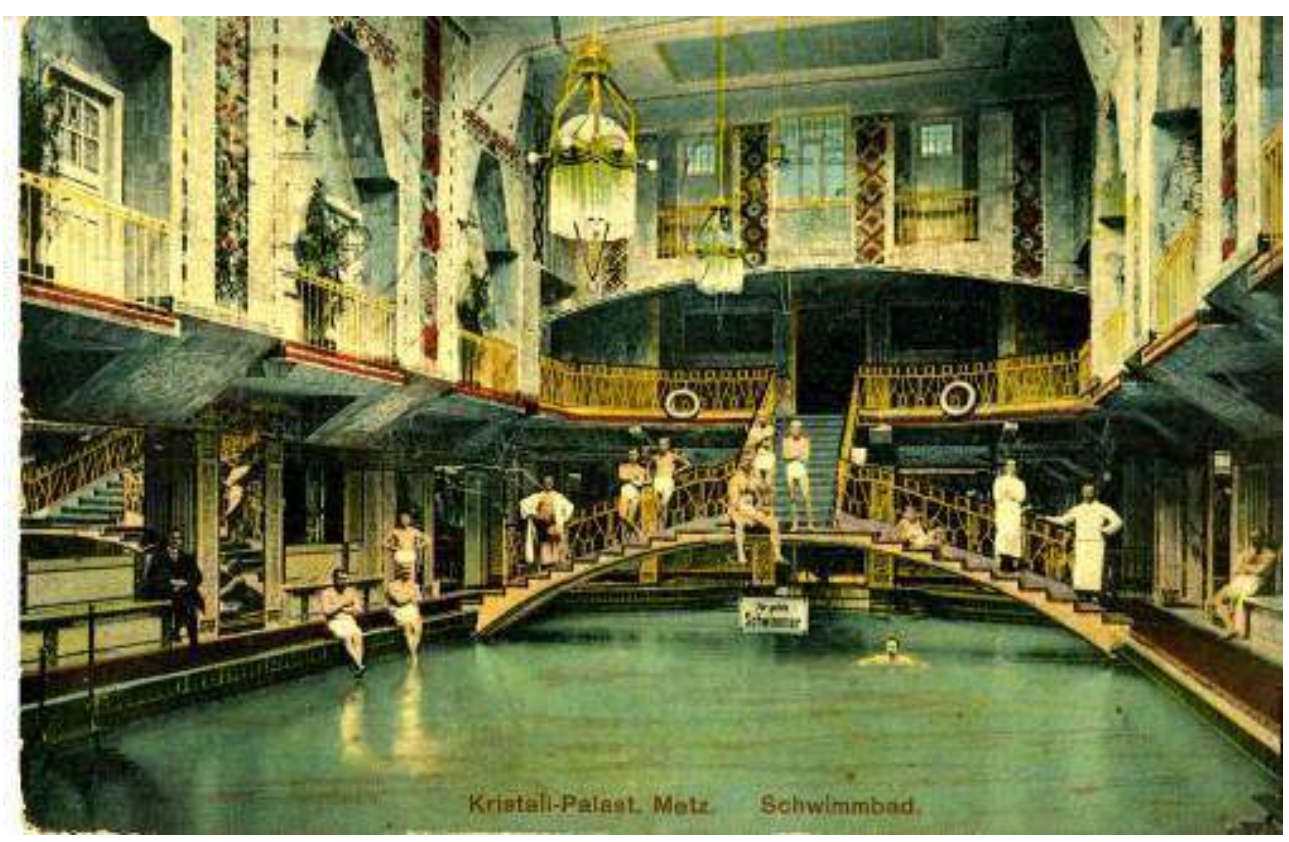

Carte postale, 1912

Collection de l'auteur

Mais la presse contemporaine ne parle-t-elle pas d'une «haute façade agrémentée de cabochons et de verrerie multicolore, d'une vaste construction constituant un ensemble problématique et d'aspect prétentieux et toc [sic]. Si les architectes et l'entrepreneur ont voulu faire quelque chose d'original, ils y ont réussi, car il y a de l'originalité dans tous les échelons du goût, du mauvais au bon ${ }^{30} »$ ? Qu'aurait dit le chroniqueur messin devant l'American Bar viennois de Loos ou la Majolica Haus de Wagner qui avaient manifestement inspiré Balassa ? ${ }^{31}$

Et que pouvait faire l'artiste devant tant d'incompréhension sinon clamer son génie et assurer la municipalité qu'il lui offrait la plus belle réalisation jamais conçue en Allemagne ?32

Le 19 février 1910 enfin, après des retards dus aux refus d'autorisation de la mairie, l'établissement était inauguré et ouvert à la folle curiosité du public. Précédée par un défilé, en fanfare, des employés, cette inauguration attira des milliers de curieux qui finirent, avec la presse, enfin unanime ${ }^{33}$, par convenir que l'établissement était le plus beau, le plus original, le plus prestigieux, le plus extraordinaire de la ville. Le plus cher aussi puisqu'il fut estimé à plus de 1200000 marks.

\section{Le naufrage}

Tel un conte des 1001 nuits se dévoilent toutes les magnificences que cache le palais de cristal $^{34}$ assurait encore la publicité le 10 mars 1910. Hélas, début avril, le commanditaire Dietsch déposait son bilan. Le 27 avril, six semaines après l'ouverture, le syndic de faillite fit fermer l'établissement, les recettes ne suffisant plus à couvrir les frais d'exploitation.

Après une première adjudication sans résultats, le 30 juin 1911, une deuxième vente, de gré à gré, mit en concurrence la maison qui avait fourni l'ameublement, un consortium 
formé par Dietsch et un consortium de Nancy. C'est ce dernier, sous le nom de Josef Barazsy ${ }^{35}$, un Français d'origine hongroise, qui l'emporta. L'établissement rouvrit en juillet 1911, à grand renfort de publicité.

Pendant la guerre de 1914-1918, le Palais de cristal fut séquestré comme bien ennemi. Barazsy n'en reprit possession qu'après la guerre. En 1920, reconnaissant que « la maison est entièrement en béton armé, les travaux de transformation sont à exécuter avec les plus grandes précautions ${ }^{36} »$, l'architecte Dedun envisagea quelques modifications. Mais c'est l'architecte Boivin qui assura son naufrage. Il fit recouvrir le bassin de natation par une dalle de béton. La piscine devint alors salle de réunion puis salle de bal, le reste de l'immeuble étant occupé par divers commerces et dépôts (ill. 6).

\section{6 : Le palais de cristal dans son environnement urbain}

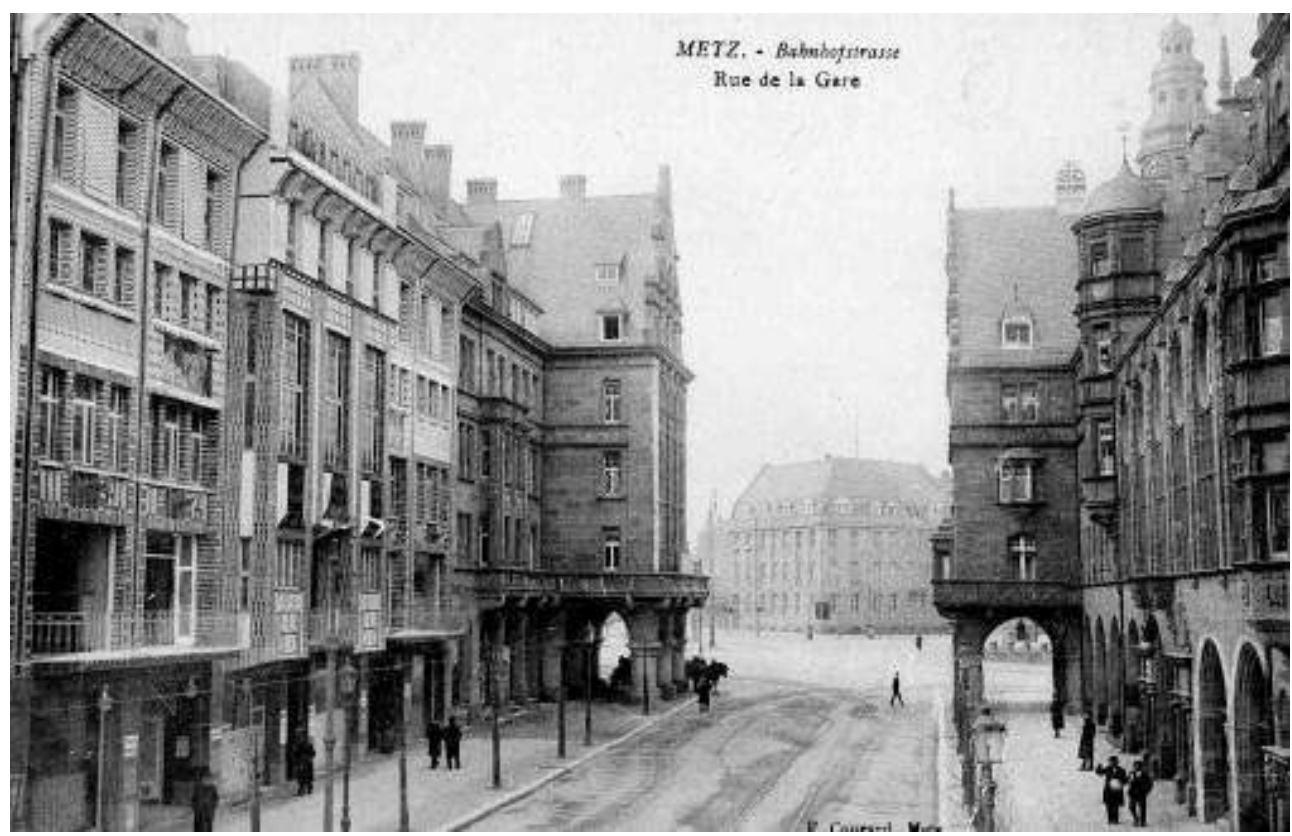

Carte postale, 1912

collection de l'auteur

Au cours de la deuxième guerre mondiale, la jeunesse hitlérienne réquisitionna toute la tuyauterie de cuivre et acheva de dénaturer le décor intérieur. Après quelques minimes dégâts subis lors de la Libération de Metz, l'immeuble fut réparé sur des fonds du ministère de la reconstruction et de l'urbanisme ${ }^{37}$.

En 1960, des plaques de cristal se détachant de la façade, le maire somma les nouveaux propriétaires de rénover cette dernière en entier ${ }^{38}$. Ce fut la fin du palais qui fut rehaussé de deux étages de bureaux et dont la façade fut recouverte de plaques de comblanchien. On ne conserva de l'intérieur que le volume de la cage d'escalier et les volées de marches. Les autres espaces servirent de bureaux à diverses associations tandis que le fonds incliné de la piscine accueillait des archives. Irréversiblement modifiée, la plus belle piscine d'Allemagne qui conjuguait tous les fantasmes et tous les arts s'en tint à la fugacité d'un rêve de cristal.

Balassa qui avait accompli le prodige de relier l'antiquité des thermes et des palais byzantins à la contemporanéité, de poursuivre la folle chimère de la transparence et d'apporter à ses semblables un nouvel art de vivre paya le prix de son audace. Considéré 
par les tribunaux correctionnels comme paranoïaque et violent ${ }^{39}$ (termes que l'on peut traduire par artiste incompris revendiquant la reconnaissance de son avant-gardisme), Balassa fut expulsé du territoire en 1912 après des épisodes assez rocambolesques. Dès lors, on perd la trace du concepteur du pharaonique palais de cristal.

Il fallut attendre 1937 pour voir se construire la première piscine municipale de Metz.

\section{NOTES}

1. "Bade-und Waschanstalt in der Kapuzinerstrasse in Metz ", Centralblatt der Bauverwaltung, 1886, $\mathrm{n}^{\circ} 48$.

2. Michèle Kuntz, Metz l'impériale, une aventure urbaine, 1850-1870, Metz, Serpenoise, 1985.

3. Christiane Pignon-Feller, Metz 1848-1918, les métamorphoses d'une ville, Metz, Serpenoise, 2006.

4. Wannenbäder, bains en baignoires; Brausebad, douches.

5. Arch. mun. Metz, 5I 208-216, Bains de rivières (1801-1870), 5I 217, Écoles de natation, (1827-1850)

6. Georg Osthoff, Die Bäder und Badeanstalten der Neuzeit, Leipzig, 1887. Rudolf Schultze, Bau und Betrieb von Volksbadeanstalten, Bonn, 1893. Leo Vetter, Moderne Bäder, Stuttgart, 1894. Et aussi proposé par un éditeur, W. Schleier, Bäder und Badeanstalten, Leipzig, s. d.

7. « Hallenbadeanstalten», Wasmuth's Baulexikon, Leipzig, 1926, t. 2/3, p. $20-27$.

8. Conseil municipal de Metz (CMM), 13 décembre 1906.

9. Les 46 îlots de la nouvelle ville se répartissaient en trois zones: zone d'immeubles serrés, à l'alignement, zone résidentielle de villas à jardinets et zone mixte. Chacune de ces zones était dotée d'un règlement spécifique.

10. CMM, 11 octobre 1906.

11. CMM, 13 décembre 1906.

12. Renseignement porté sur la «Fiche domiciliaire » de Johann Balassa qui reste hélas muette sur sa formation.

13. CMM, 7 novembre et 5 décembre 1907.

14. Arch. mun. Metz, Règlement sur les constructions pour la ville de Metz du 1er février 1903.

15. Articles $15,29,36,37,39,41$ du règlement urbain.

16. Lettres à en-têtes de la société.

17. Arch. mun. Metz 1M 56, lettre du 19 août 1909.

18. Le Lorrain, mars 1908

19. La surabondante correspondance de Balassa et de Dietsch avec la municipalité ne laisse aucun doute sur les obstacles que dut surmonter l'entreprise. La presse germanophone et francophone ne fit qu'exacerber les débats.

20. Sur le système des pilotis à chasse d'eau employés à la poste de fret, à la gare et à la poste centrale de Metz, voir Deutsche Bauzeitung, 1906, p. 600.

21. Arch. mun. Metz, 1M 56, correspondance de Balassa avec la municipalité.

22. Cette information donnée par Le Lorrain le $1^{\text {er }}$ avril fut confirmée deux jours après avec l'assurance qu'il ne s'agissait pas d'un poisson d'avril ! 
23. Nous proposons les traductions littérales, plus savoureuses: Fingernägelschneider, coupeurs d'ongles, Hühneraugenoperator, extracteur de cors aux pieds, Zahnreiniger, détartreur de dents.

24. Le Lorrain, publicité, février 1910.

25. Les ouvrages allemands sur les établissements de bains déclinent également toutes les possibilités.

26. Rappelant les origines austro-hongroises de Balassa.

27. Arch. mun. Metz, 1M 56, lettre du 25 mai 1909.

28. Arch. mun. Metz, $1 \mathrm{M} 56$, Balassa fait état de voyages à Vienne, en Allemagne et en France au cours de la construction de son palais.

29. Il en reste.

30. Le Messin, 9 février 1910.

31. Une des cellules commerciale du palais était également réservée à un American Bar.

32. Arch. mun. Metz 1M 56, lettres de juin 1909.

33. C'est surtout le Zillertal qui fascina les Messins et la presse francophone et germanophone.

34. Le Lorrain, 10 mars 1910.

35. Français d'origine hongroise demeurant à Pagny sur Moselle, frontière entre la France et la Moselle annexée.

36. Arch. mun. Metz, $1 \mathrm{M} 56$, liasse 2.

37. Archives dép. Moselle, fonds Bouchard.

38. Arch. mun. Metz, $1 \mathrm{M} 56$, liasse 3.

39. Le Lorrain, 1911, pour les procès de Balassa et ses suites.

\section{RÉSUMÉS}

Au cours de l'annexion de la Moselle au deuxième Reich allemand, un jeune architecte austrohongrois, Johann Balassa, se substitue à la municipalité et entreprend de construire un établissement balnéaire (1907-1910) totalement nouveau dans la nouvelle ville. Au grand scandale des édiles et s'opposant à la réglementation urbaine et esthétique en vigueur, il outrepasse le programme imparti et, sur le modèle des thermes antiques et des établissements des villes d'eau du XIX siècle, il donne à Metz un exemple de modernité technique, de décor inédit, de culte ludique du corps et de philosophie hédoniste. Dans le premier bâtiment entièrement en béton armé de Metz, aidé par l'entrepreneur et maître d'ouvrage spécialiste du béton armé Nicolas Dietsch, il hisse le bassin de natation (autoportant) au deuxième étage et pare les bassins, les parois et la façade de plaques de cristal et de mosaïques conférant ainsi à l'établissement le nom de palais de cristal. Il offre aux Messins tous les soins du corps et les délassements modernes et conviviaux de l'époque (cinémas, restaurants automatiques, salles de jeux...). Il puise le décor de stuc, de verre et de ferronnerie chez les meilleurs maîtres de la Sécession viennoise et des Wiener Werkstätte. Après quelques semaines d'exploitation, « le plus bel établissement de ce genre en Allemagne " sombra dans une faillite retentissante et au cours des vicissitudes de l'histoire de la ville fut, hélas, totalement dénaturé. 
While the department of Moselle was being annexed to the German Second Reich, a young Austro-Hungarian architect, Johann Balassa, substituted himself for the local authority and undertook to have brand new bath houses built (1907-1910) in the New City. He went far beyond the agreed program, which scandalized the town councillors and went against the urban and aesthetic regulations in force, and, as he modelled them on the antique thermal baths and the $\mathrm{XIX}^{\text {th }}$ century spa resorts, he brought the town of Metz a model of technical modernity, of an original decoration, of an entertaining body worship and of a hedonist philosophy. In this building that was the first to be all made of reinforced concrete in Metz, as he was helped by Nicolas Dietsch as the contractor and the commissioning specialist in reinforced concrete, he set the main pool up to the second floor and had the pools, the sides and the façade covered up with crystal and mosaic plates, thus naming the establishment the "palais de cristal". He offered the inhabitants of Metz all the body care and the modern convivial relaxations of the time (cinemas, automats, gaming rooms...). As for the stucco, glass and iron decoration, he inspired from the best masters of the Viennese Secession and of the Wiener Werkstätte. After a few weeks of running, "the most beautiful place of this kind in Germany" went a spectacular bankrupt and was, alas, completely altered throughout the vicissitudes of the town history.

Während der Annektierung der Mosel durch das Deutsche Kaiserreich übernimmt Johann Balassa, ein junger österreichisch-ungarischer Architekt, eigenmächtig die Aufgabe der Stadtverwaltung, in der Stadt Metz eine ganz neuartige Badeanstalt zu bauen (1907-1910). Zum großen Ärger der Stadtväter und entgegen der bestehenden urbanen und ästhetischen Regeln sprengt er den ihm zugestandenen Rahmen. Er setzt sich zwar als Modell die Thermen der Antike und die Badeanstalten der Kurorte des 19. Jahrhunderts, bietet aber in Metz eine beispielhafte Mischung aus technischer Modernität und ungewohntem Dekor, die auf spielerische Art den Kult des Körpers und die hedonistische Philosophie zelebriert. Das erste Gebäude wird aus Metzer Stahlbeton von dem Bauunternehmer und Baumeister Nicolas Dietsch gebaut. Dank dessen fortgeschrittener Technik wird das selbsttragende Schwimmbecken in die zweite Etage gehoben. Becken sowie Wände und Fassade werden mit Kristall- und Mosaikscheiben geschmückt, was der Anstalt seinen Namen Kristallpalast verleiht. Die Ornamente aus Gips, Glas und Kunstschmiedeeisen werden bei den besten Meistern der Wiener Sezession und der Wiener Werkstätte ausgesucht. Die Anstalt bietet den Metzern außerdem alles mögliche für Körperpflege an, so wie jede moderne und benutzerfreundliche Unterhaltung dieser Zeit, unter anderem Kinos, Automatenrestaurants, Spielsäle... Schon nach ein paar Wochen Betrieb aber versinkt "die schönste Anstalt dieser Art in Deutschland" in einem Bankrott ohne Beispiel und wird im Verlauf der wechselvollen Stadtgeschichte leider völlig unkenntlich gemacht.

\section{INDEX}

Index chronologique : XIXe siècle, XXe siècle, époque contemporaine

Mots-clés : piscine

Keywords : swimming pool

Schlüsselwörter : Schwimmbad 


\section{AUTEUR}

\section{CHRISTIANE PIGNON-FELLER}

Christiane Pignon-Feller, née en 1940, après une carrière de professeur de lettres, reprit des études en histoire de l'art à l'Université de Nancy II. Dès son mémoire de maîtrise, en 1992, elle se tourna vers l'étude de la ville de Metz au XIX ${ }^{\mathrm{e}}$ siècle (Quand l'ornement n'était pas un crime, les arts décoratifs à Metz au XIX ${ }^{e}$ siècle, université Nancy II). L'année suivante, elle obtint un DEA (La petite illusion, les petits théâtres en images de Metz ) et se lança alors dans un travail de thèse sur l'architecture et le décor à Metz dans la seconde moitié du XIX ${ }^{\mathrm{e}}$ siècle, sous la direction du professeur François Pupil, soutenue en 1999 (Du Second Empire au deuxième Reich, de la vieille ville à la nouvelle ville, urbanisme, architecture, décor, Metz, 1848-1918). Depuis 1999, elle est chargée de missions d'étude et d'inventaire pour la ville de Metz, l'inventaire général de Lorraine, le conseil général de Moselle et participe à de nombreux colloques et publications sur l'architecture, les arts décoratifs et les arts graphiques. Publications principales : Metz 1848-1918, les métamorphoses d'une ville, Metz : éditions Serpenoise, 2006. Moselle XVII ${ }^{e}-X X^{e}$ siècle, Architecture protestante, Metz : conseil général de la Moselle / éditions Serpenoise, 2006. Adresse électronique :

feller.christiane@neuf.fr 